\title{
Correlação entre orientação molecular, cristalinidade e permeabilidade de filmes biaxialmente orientados de polietileno linear de baixa densidade
}

\author{
Correlation between molecular orientation, \\ crystallinity, and permeability of biaxially \\ oriented linear low-density \\ polyethylene films
}

\author{
${ }^{1}$ Departamento de Engenharia de Materiais, Escola de Engenharia de São Carlos, Universidade de São Paulo CEP:15566- \\ 590 São Carlos, SP. \\ ${ }^{2}$ Instituto Federal do Amazonas, CEP69020-120 Manaus, AM. \\ e-mail: mesquita.barros@ifam.edu.br; marciacb@sc.usp.br
}

\begin{abstract}
RESUMO
Embora diferentes segmentos do mercado de filmes poliméricos possuam diferentes exigências de desempenho, as propriedades dos filmes dependem da arquitetura molecular, microestrutura, orientação molecular e cristalinidade, desenvolvidas em função do tipo de maquinário utilizado e das condições do processamento. Portanto, o conhecimento das microestruturas desenvolvidas é de grande interesse científico e tecnológico. Neste trabalho foi estudada a correlação entre orientação molecular, cristalinidade e propriedades de permeabilidade, ao vapor de água e ao oxigênio, de filmes biaxialmente orientados de polietileno linear de baixa densidade (PELBD), obtidos pelo processo de extrusão plana e estirados com diferentes razões de estiramento. O estudo da orientação molecular revelou a mudança da estrutura morfológica das cadeias da fração cristalina dos filmes submetidos a maiores graus de estiramento. Resultados do grau de cristalinidade dos filmes de PELBD evidenciaram uma tendência de aumento da cristalinidade com o aumento da orientação das cadeias poliméricas, devido ao estiramento. Resultados de propriedades de barreira mostraram que o aumento do grau de estiramento possui efeito moderado na diminuição da permeabilidade ao oxigênio e ao vapor de água dos filmes PELBD, e que esse comportamento está relacionado ao grau de estiramento e cristalinidade dos filmes.
\end{abstract}

Palavras-chave: Polietileno linear de baixa densidade, Orientação molecular, Permeabilidade, Cristalinidade.

\begin{abstract}
Although different polymeric film market segments have different performance requirements, the properties of the films depend upon the molecular architecture, microstructure, molecular orientation and crystallinity, developed according to the chosen processing tools and conditions. Therefore, knowledge of developed microstructure is of great scientific and technological interest. In this study, it was studied the correlation between molecular orientation, crystallinity and water vapor and oxygen permeability properties of biaxially oriented linear low-density polyethylene (LLDPE) films obtained by planar extrusion and stretched by different stretch rations. The molecular orientation study revealed morphological structure changing of chains in the crystalline fraction of the films submitted to higher degrees of stretching. Results of degree of crystallinity of LLDPE films evidenced a tendency to increase crystallinity with increasing orientation of the polymer chains, due to stretching. Results of barrier properties showed the increase of the degree of stretching has a moderate effect on the decrease of permeability to oxygen and water vapor of the LLDPE films, and that this behavior is related to the degree of stretching and crystallinity of the films.
\end{abstract}

Keywords:Linear low-density polyethylene, Molecular orientation, Permeability, Crystallinity.

\section{INTRODUÇÃO}


Os segmentos do mercado para filmes poliméricos continuam apresentando expressivo crescimento mundial. Simultaneamente, os transformadores e fornecedores de filmes procuram soluções que ofereçam redução de custo, alta produtividade, boa processabilidade e capacidade de automação da manufatura, além de exigências de desempenho, como resistência mecânica, estabilidade estrutural, boas propriedades de permeabilidade e ópticas, entre várias outras, de acordo com a aplicação final do filme. Resinas de polietileno linear de baixa densidade (PELBD) são usadas principalmente na produção de filmes, devido às suas inúmeras vantagens tais como boa resistência ao rasgamento e ao impacto. Resinas de PELBD possuem densidade entre 0,91$0,94 \mathrm{~g} / \mathrm{cm}^{3}$ e são copolímeros de etileno obtidos a partir da polimerização de eteno e de $\alpha$-olefinas, como: 1 buteno, 1-hexeno, 1-octeno ou propeno, incorporadas na cadeia polimérica. A microestrutura da cadeia dos copolímeros de etileno/ $\alpha$-olefinas depende do tipo e da distribuição do comonômero usado, do teor de ramificações e do peso molecular dos polímeros. Esses parâmetros influenciam as propriedades físicas do produto final, pois atuam diretamente na cristalinidade e na morfologia semicristalina [1]. Com cadeias lineares de baixo grau de ramificações curtas, o PELBD cristaliza em lamelas mais ordenadas e mais espessas do que o polietileno de baixa densidade (PEBD). Conseqüentemente, o PELBD apresenta melhores propriedades mecânicas, maior temperatura de fusão, na faixa de 120 a $130^{\circ} \mathrm{C}$, maior cristalinidade, variando de 22 a $55 \%$, e boas características ópticas quando comparado ao PEBD [2]. Por outro lado, a maior resistência ao cisalhamento e a maior susceptibilidade à fratura do fundido fazem com que o processamento do PELBD seja mais difícil em comparação com o do PEBD [3]. O PELBD é um termoplástico com elevada capacidade de selagem a quente, sendo muito utilizado em embalagens, substituindo o PEBD em várias aplicações. A extrusão de filmes planos de PELBD fornece produtos para serem utilizados em plástico bolha, fraldas descartáveis, absorventes higiênicos, lonas, sacarias em geral, entre outras aplicações.

A caracterização dos efeitos dos processos de fabricação de materiais poliméricos é um campo de interesse na área da ciência e engenharia de polímeros. As principais caracterizações são a determinação da orientação molecular imposta por processos deformacionais e a inter-relação entre a orientação molecular, a estrutura morfológica e as propriedades físicas [3]. A orientação molecular é um fenômeno único dos polímeros, modifica as propriedades mecânicas, térmicas, de barreira, ópticas e até mesmo a resistência aos produtos químicos, à irradiação ultravioleta, entre outras, e é dependente das condições experimentais utilizadas no processamento, assim como das características intrínsecas do material [4]. Por definição, a orientação molecular em um polímero semicristalino descreve as diversas possibilidades de alinhamento dos eixos -a, -b e -c da molécula em relação a um referencial externo, como a direção normal (DN), direção transversal (DT), e direção do escoamento/estiramento (direção da máquina, DM). Entretanto sabe-se que qualquer orientação induzida em polímeros nunca é perfeita, isto é, existe uma distribuição de orientação [5, 6]. O estiramento de filmes poliméricos é usualmente efetuado para melhorar as propriedades mecânicas dos mesmos, e, sob determinadas condições, também pode levar a uma melhora nas propriedades de barreira e ópticas, por exemplo. Portanto, a orientação molecular é em geral desejável para filmes e fibras, pois ocorre considerável melhoria das propriedades na direção do estiramento [4]. Estudos de orientação de filmes ou fibras de polímeros semicristalinos têm demonstrado que as cadeias poliméricas nas fases cristalinas se orientam em primeiro lugar, ficando as cadeias das regiões amorfas, inicialmente com uma ligeira inclinação, um pouco mais perpendiculares à direção de estiramento, se orientando a seguir, porém com um grau de orientação mais baixo do que as cadeias das fases cristalinas. Sendo assim, o polímero orientado adquire maior grau de cristalinidade ao final do processo de orientação [4, 7, 8]. A orientação molecular de polímeros semicristalinos pode ser investigada por vários métodos laboratoriais. Recentemente, melhorias em fontes de luz laser, dispositivos ópticos e detectores têm promovido o uso da técnica de espectroscopia Raman, devido principalmente ao seu curto tempo de aquisição, permitindo assim que as medições sejam realizadas in situ $[9,10]$. Neste estudo, a avaliação da orientação molecular dos filmes de PELBD foi efetuada pelo uso da técnica de espectroscopia de absorção de infravermelho polarizado (Polarized Infrared Absorption Spectroscopy), sendo essa uma técnica bastante versátil, de rápida execução e interpretação, não-destrutiva e que não requer preparação elaborada das amostras.

A cristalinidade de polímeros depende da estrutura química, do peso molecular e do tratamento físico, incluindo temperatura, tempo e forças às quais o material foi submetido. No caso de filmes biaxialmente orientados de polietileno, as morfologias de estruturas nucleadas denominadas Keller-Machin I e II foram propostas por Keller e Machin em 1967 [11]. De acordo com esse modelo de estruturas, duas principais estruturas podem ocorrer no processo de cristalização de acordo com a tensão aplicada no processamento desses filmes. Baixos graus de tensão, freqüentemente, resultam na estrutura Keller-Machin I, na qual o crescimento das lamelas se dá a partir do núcleo de fibrilas sob a forma de fitas torcidas, com a direção de crescimento paralela ao eixo-b da célula unitária cristalina do polietileno e uma orientação preferencial do eixo-a paralela à DM. Altos graus de estiramento resultam na estrutura Keller-Machin II, na qual as lamelas planas crescem 
radialmente e se estendem diretamente para o exterior, sem torção, e as cadeias regularmente dobradas dentro das lamelas permanecem orientadas na direção da máquina, ou seja, o eixo-c da célula unitária cristalina do polietileno se orienta preferencialmente na DM.

Uma grande aplicação de interesse comercial de filmes de polietileno está na obtenção de embalagens com melhores propriedades de barreira. Os polietilenos são, por natureza, apolares e excelentes barreiras para a umidade e vapor de água, mas podem ser pobres quando se trata de barreira ao oxigênio [12, 13]. São várias as variáveis do polímero que afetam as propriedades de barreira, sendo a área, a espessura e as características moleculares do polímero, tais como: grau de polaridade, rigidez e grau de compactação das cadeias, advindos da simetria molecular, grau de cristalinidade ou orientação molecular, grau de ligação ou atração entre as cadeias, consideradas as principais [14].

Nesse contexto, fica evidente que os fabricantes de filmes poliméricos possuem patente demanda tecnológica do conhecimento de como as diversas propriedades dos filmes variam em função das condições de processo e do tipo de maquinário utilizados. Baseados nessa demanda, no presente trabalho foi avaliada a influência do grau de estiramento na orientação molecular, na cristalinidade e nas propriedades de barreira, permeabilidade ao vapor de água e ao oxigênio, dos filmes de PELBD obtidos por extrusão plana. As caracterizações das propriedades foram realizadas pelo uso de técnicas simples, disponíveis e conhecidas. Esperase que as informações obtidas sobre cristalinidade, permeabilidade ao oxigênio e permeabilidade ao vapor de água em função da orientação molecular caracterizada, melhorem o entendimento do processo de estiramento e seus produtos, no caso filmes biorientados de PELBD, visando permitir ajustes de processo de acordo com usos finais específicos do produto.

\section{MATERIAL E MÉTODOS}

\subsection{Obtenção dos filmes de PELBD por extrusão e estiramento biaxial}

Os filmes de PELBD estudados foram produzidos por extrusão de filme plano em extrusora com rosca de 63 $\mathrm{mm}$ de diâmetro, razão comprimento (L/D) igual a 24, abertura da matriz plana de 1,5 mm, e variadas razões de estiramento DM:DT, seguido de tratamento térmico a $90^{\circ} \mathrm{C}$ e resfriado a temperatura ambiente. Os filmes foram processados com razões de estiramento DM:DT de 2:1, 3:1; 3,5:1; 4:1 e 4,5:1, os quais são denominados nesse trabalho por PE-or (filme original, comercialmente fabricado); PE-3; PE-3,5, PE-4 e PE-4,5, respectivamente, e foram produzidos pela empresa Polysur.

O filme não orientado de PELBD (isotrópico), denominado PE-no, foi preparado no laboratório a partir do aquecimento do filme PE-or a $150^{\circ} \mathrm{C}$, moldado entre filmes de Kapton ${ }^{\circledR}$ e resfriado em água fria. Todos os filmes foram produzidos a partir da resina de polietileno comercial sintetizada por copolimerização do etileno com comonômero de 1-octeno que, de acordo com os dados técnicos do fabricante Dow Chemical, possui índice de fluidez de $3,5 \mathrm{~g} / 10 \mathrm{~min}$ a $190^{\circ} \mathrm{C}$ e $2,16 \mathrm{Kg}$, densidade de $0,915 \mathrm{~g} / \mathrm{cm}^{3}$ e temperatura de fusão $\left(\mathrm{T}_{\mathrm{m}}\right)$ de $123^{\circ} \mathrm{C}$. A Tabela 1 lista os filmes estudados, suas razões de estiramento e espessura média.

Tabela 1: Razões de estiramento biaxial e espessura dos filmes estudados.

\begin{tabular}{l|l|l}
\hline AMOSTRA & $\begin{array}{l}\text { RAZÃO DE ESTIRAMENTO } \\
\text { DM:DT }\end{array}$ & $\begin{array}{l}\text { ESPESSURA } \\
(\boldsymbol{\mu} \mathbf{m})\end{array}$ \\
\hline PE-or & $2: 1$ & $50 \pm 2$ \\
\hline PE-3 & $3: 1$ & $50 \pm 3$ \\
\hline PE-3,5 & $3,5: 1$ & $50 \pm 3$ \\
\hline PE-4 & $4: 1$ & $49 \pm 4$ \\
\hline PE-4,5 & $4,5: 1$ & $50 \pm 2$ \\
\hline PE-no & - & $48 \pm 7$ \\
\hline
\end{tabular}

\subsection{Métodos de caracterização}

A espessura média dos filmes de PELBD (ou dos filmes PE's) foi determinada por medidas realizadas em 3 diferentes pontos dos filmes com o uso de um micrômetro da marca Zaas com precisão de 0-25x0,01mm. Os resultados de espessura média foram listados na Tabela 1.

As medidas de orientação molecular, por dicroísmo no infravermelho polarizado, foram realizadas pela obtenção do espectro de absorbância, na faixa de comprimento de onda da radiação infravermelha entre 
800 e $600 \mathrm{~cm}^{-1}$ com resolução de $0,5 \mathrm{~cm}^{-1}$, e com o polarizador posicionado a $0^{\circ}$, para medidas na direção paralela, e a $90^{\circ}$, para medidas na direção perpendicular, em relação à direção de referência dos filmes. A direção da máquina, DM, foi adotada como direção de referência. As medidas foram realizadas em triplicata no espectrofotômetro de infravermelho (IV) da marca Varian modelo 640-IR, equipado com um polarizador de grade de tálio, da marca Specac, modelo KRS-5.A orientação molecular dos filmes de PELBD foi avaliada através do cálculo da razão dicróica (RD) para as bandas de absorção da radiação infravermelha de natureza dicróica em $730 \mathrm{~cm}^{-1}\left(\mathrm{RD}_{730}\right)$ e $720 \mathrm{~cm}^{-1}\left(\mathrm{RD}_{720}\right)$, de acordo com as equações 1 e 2 , respectivamente. A área das bandas de absorção medidas com a radição polarizada paralela $\left(\mathrm{A}_{/ / 730} \mathrm{e} \mathrm{A}_{/ / 720}\right)$ e perpendicular $\left(\mathrm{A}_{\perp_{730}}\right.$ $\mathrm{eA}_{\perp_{720}}$ ) em relação ao referencial do filme, foram calculadas por deconvolução matemática.

$$
\begin{aligned}
& R D_{730}=\frac{A_{/ / 730}}{A_{\perp 730}} \\
& R D_{720}=\frac{A_{/ / 720}}{A_{\perp 720}}
\end{aligned}
$$

As análises de difração de raios-X de altos ângulos (WAXD) dos filmes foram realizadas pelo método de reflexão utilizando-se difratômetroRotaflex, modelo RU200B, câmara multipurpose, com radiação CuK $\alpha$ de comprimento de onda $\lambda=1,54056 \AA$, operando a $40 \mathrm{kV}$ e $80 \mathrm{~mA}$, com $2 \theta$ variando entre 3 e $40^{\circ}$, a uma taxa de $1 \%$ min. O grau de cristalinidade $(\mathrm{Xc})$ das amostras foi obtido através da deconvolução matemática dos picos dos difratogramas e calculado pela relação simples entre as áreas dos picos cristalinos $\left(\mathrm{A}_{\text {crist }}\right)$ e o halo amorfo $\left(\mathrm{A}_{\text {amorfo }}\right)$, como mostra a equação 3.

$$
X c=\frac{\sum \text { Acrist }}{\sum \text { Acrist }+\sum \text { Aamorfo }} \cdot 100
$$

Medidas de permeação ao oxigênio foram realizadas pelo método coulométrico, de acordo com a norma ASTM D3985-05 [15] utilizando-se o equipamento Ox-Tran ${ }^{\circledR}$, modelo 2/20 da Mocon ${ }^{\circledR}$. As amostras dos filmes foram inicialmente condicionadas em ambiente desumidificado a $23^{\circ} \mathrm{C}$ por 24 horas. Posteriormente, as amostras foram colocadas em máscaras de alumínio com área efetiva de permeação de $5 \mathrm{~cm}^{2}$. Durante o ensaio o fluxo de oxigênio foi de $10 \mathrm{~cm}^{3} / \mathrm{min}$, utilizando o modo de convergência por ciclos. O oxigênio utilizado foi oxigênio com $99,999 \%$ de pureza e o gás de arraste usado consistiu de uma mistura $\mathrm{N}_{2} / \mathrm{H}_{2}(98 / 2)$ padrão primário ABNT 218-2. As medidas foram então realizadas a cada 30 minutos, até que não houvesse mais variação do valor medido para medidas realizadas em 3 períodos subseqüentes, e o tempo de condicionamento foi de 2 horas. A taxa de permeabilidade ao oxigênio $\left(\mathrm{TPO}_{2}\right)$ é dada em $\mathrm{cm}^{3} / \mathrm{m}^{2}$.dia. A permeabilidade ao oxigênio $\left(\mathrm{PO}_{2}\right)$ em $\mathrm{cm}^{3} \cdot \mathrm{cm} / \mathrm{m}^{2} \cdot$ dia.atm $\quad\left(\right.$ conversão: $1 \mathrm{~cm}^{3} \cdot \mathrm{cm} / \mathrm{m}^{2} \cdot$ dia.atm $=$ $6,57 \times 10^{10} \mathrm{~cm}^{3} . \mathrm{cm} / \mathrm{cm}^{2}$.s.cmHg) é calculada pela equação 4 .

$$
\mathrm{PO}_{2}=\frac{\mathrm{TPO}_{2}}{\mathrm{PpO}_{2}} \cdot e
$$

onde $e$ é a espessura média do filme (cm) e $\mathrm{P}_{\mathrm{O} 2}$ é a pressão parcial do oxigênio na câmara de teste (1atm), sendo que a pressão parcial de oxigênio na câmara de gás de arraste é considerada nula.

Medidas de permeação ao vapor de água foram realizadas de acordo com a norma ASTM F1249-13 [16]. Durante o ensaio as amostras foram pesadas a cada 24 horas, por um período de 10 dias, em balança analítica. A umidade relativa do ar e a temperatura $\left(23 \pm 1^{\circ} \mathrm{C}\right)$ do ambiente foram controladas pela climatização da sala. Os valores de permeabilidade a vapor de água $\left(\mathrm{P}_{\mathrm{WV}}\right) \mathrm{em} \mathrm{cm}^{3} \cdot \mathrm{cm} / \mathrm{cm}^{2} \cdot h$.barforam calculados de acordo com as equações 5 e 6 .

$$
\begin{aligned}
& P_{W V}=\frac{W V T}{S\left(R_{1}-R_{2}\right)} \cdot e \\
& W V T=\frac{G}{T \cdot A_{p}}
\end{aligned}
$$

onde WVT é a taxa de transmissão de vapor de água $\left(\mathrm{g} / \mathrm{cm}^{2} . \mathrm{h}\right)$; S é a pressão de saturação do vapor de água na temperatura do ensaio (0,028 bar) [17]; $\mathrm{R}_{1}$ e $\mathrm{R}_{2}$ são as umidades relativas do ar em cada uma das faces da amostra, 1 e 0,22\%, respectivamente; G é a variação de massa (g); t é o tempo (h); e $A_{p}$ é a área de permeação $\left(\mathrm{cm}^{2}\right)$. 


\section{RESULTADOS E DISCUSSÕES}

Os filmes de PELBD foram caracterizados quanto a sua microestrutura, orientação molecular, grau de cristalinidade e propriedade de permeação ao oxigênio e ao vapor de água. A Figura 1 apresenta alguns dos espectros obtidos por espectroscopia no infravermelho (IV), com polarizações paralela (linha tracejada) e perpendicular (linha cheia) em relação ao referencial externo (adotada a direção da máquina, DM), e na faixa de comprimento de onda de 770 a $670 \mathrm{~cm}^{-1}$, para as amostras estudadas.

A banda de absorção do polietileno a $730 \mathrm{~cm}^{-1}$ é atribuída à vibração em balanço do grupo $\mathrm{CH}_{2}$ presente na fase cristalina do polímero e apresenta dicroísmo paralelo ao eixo-a da célula unitária do PE. A banda de absorção a $720 \mathrm{~cm}^{-1}$ é atribuída à vibração de sacudida, contendo componentes devido às fases cristalinas e amorfas do polímero, sendo que a componente cristalina desta banda de absorção apresenta dicroísmo paralelo ao eixo-b (eixo de crescimento) da cadeia de polietileno [5]. Os valores médios de razão dicróica, $\mathrm{RD}_{730}$ e $\mathrm{RD}_{720}$, para os espectros obtidos foram calculados a partir das equações 1 e 2 e apresentaram desvio médio de $\pm 2 \%$. Para facilitar a visualização da influência do grau de estiramento dado na direção DM sobre a orientação molecular dos eixos-a e -b nos filmes de PE estudados, foram plotadas as curvas dos valores médios de $\mathrm{RD}_{730}$ e $\mathrm{RD}_{720}$ versus filme e seu estiramento, como mostra a Figura 2.
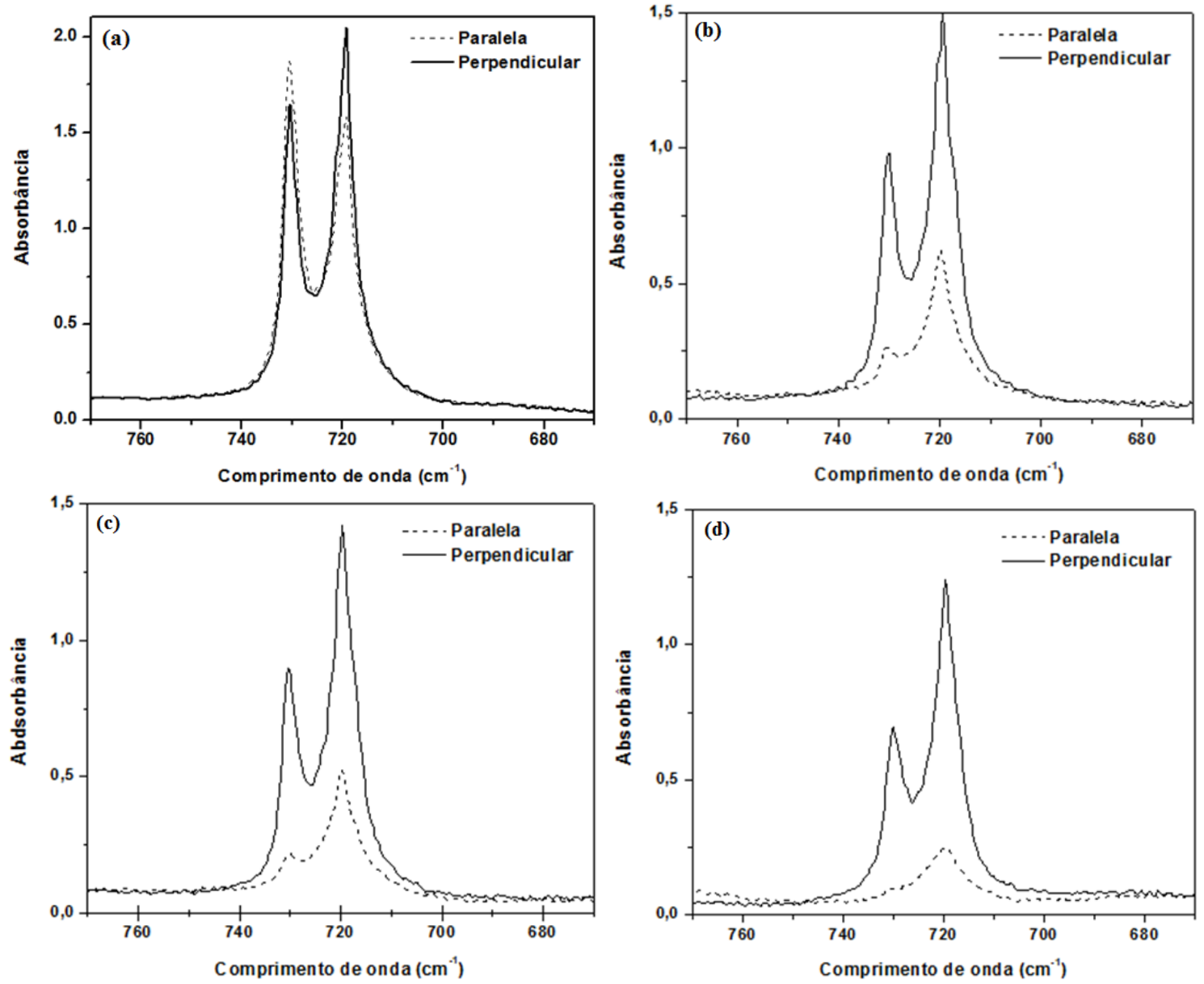

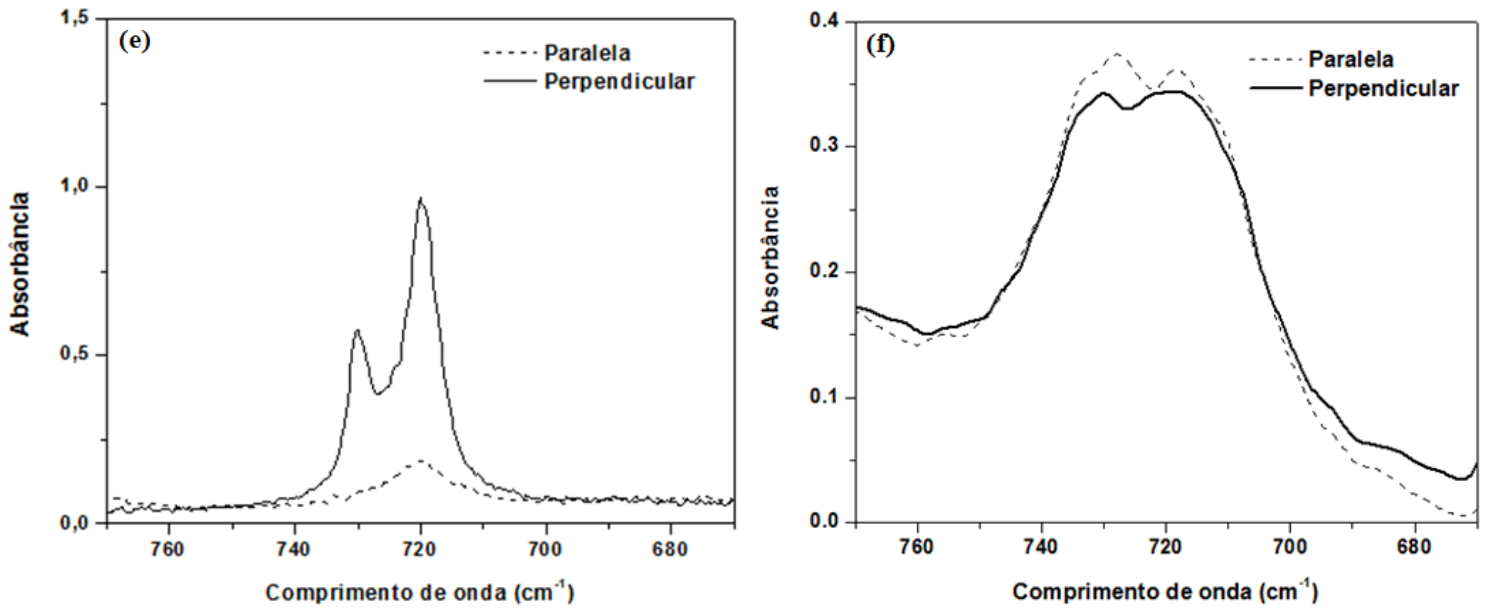

Figura 1: Espectros de IV com polarização paralela e perpendicular dos filmes (a) PE-or; (b) PE-3; (c) PE-3,5; (d) PE-4; (e) PE-4,5 e (f) PE-no.

Os resultados mostram que no filme de PE-or a orientação preferencial da fase cristalina do polietileno é dada com o eixo-a da célula unitária orientado na direção paralela em relação a direção referencial, ou seja,ao longo da direção da máquina (DM), enquanto o eixo-b se orienta preferencialmente na direção perpendicular à DM. Sendo assim, pode-se afirmar que os eixos-b e -c estão distribuídos ao longo das direções transversal (DT) e normal (DN) do filme. Esse arranjo é conhecido na literatura por Keller-Machin I ou textura-a, sendo resultante de estruturas altamente ordenadas com lamelas empilhadas chamadas cilindritos [11].

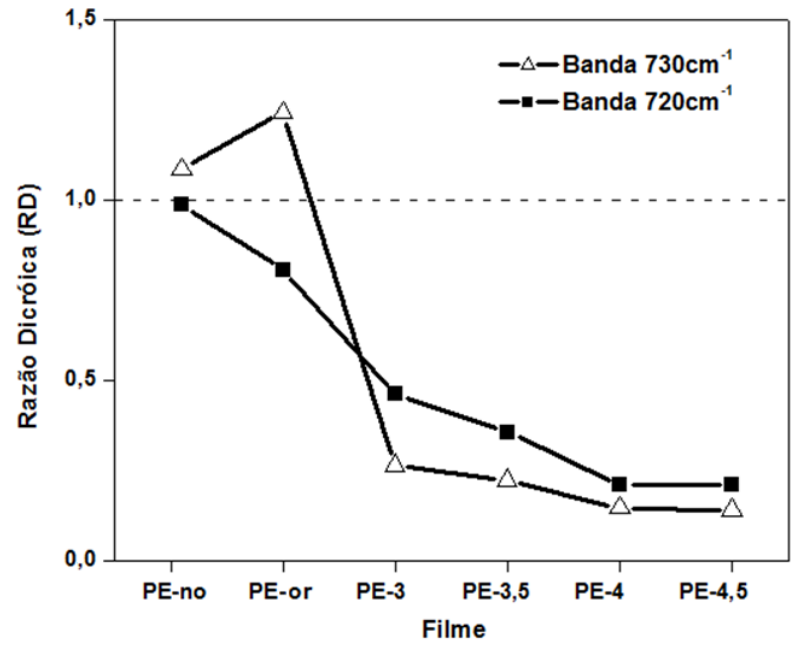

Figura 2: Variação da $\mathrm{RD}_{730}$ e $\mathrm{RD}_{720}$ para os filmes de PE.

Os filmes PE-3, PE-3,5, PE-4 e PE-4,5 apresentaram comportamento semelhante em termos de tipo de orientação, sendo que quanto maior o grau de estiramento na DM, maior o alinhamento dos eixos segundo a estrutura Keller-Machin II (textura-c). Para os maiores estiramentos na DM estudados, ou seja, 4 e 4,5, não foi observada alteração significativa nos valores de $\mathrm{RD}_{730}$ e $\mathrm{RD}_{720}$. Nos espectros de IR polarizados obtidos para o filme de polietileno não orientado (PE-no, Figura 1(f)) observa-se que as bandas possuem praticamente a mesma intensidade independente da polarização, indicando a não orientação preferencial dos eixos da célula unitária do polietileno, pois o tratamento térmico realizado na amostra destroi qualquer orientação gerada no processamento do filme. Para o filme PE-no, os valores calculados de $R_{730}$ e $R_{720}$ são 1,084 e 0,986, respectivamente, ou seja próximos à unidade, indicando que as cadeias poliméricas não apresentam orientação espacial preferencial.

A orientação molecular e a morfologia lamelar de filmes de polietileno têm sido extensivamente estudadas [5, 6, 11, 18-21] e uma detalhada caracterização de filmes de PELBD foi dada por Cole e Ajji [5]. No presente estudo, o filme PE-or apresentou típica cristalização de estrutura Keller-Machin I, resultante de bai- 
xa tensão aplicada no processo de estiramento do filme, enquanto os demais filmes apresentaram cristalização de estrutura Keller-Machin II, resultante de altos graus de estiramento. É consenso na literatura que a estrutura Keller-Machin I, indicando a orientação do eixo-a paralela à DM, é a mais comumente encontrada em filmes biaxiais de PELBD [22], PEBD [23, 24] e PEAD [18, 25]. Schlotter e Furlan [19] descreveram a morfologia de filmes biorientados de polietileno, na qual a orientação dos eixos-b é dada na DN e dos eixos-a e -c no plano do filme, sendo formada com a preferencial nucleação na superfície do filme e crescimento do cristal na direção da espessura do filme.

Outros trabalhos [21, 26] descrevem que filmes de PELBD e de PEBD apresentam fraca orientação do eixo-b na direção paralela à DM (à direção do estiramento) a baixas tensões, e acrescentam que os esferulitos de PELBD não estão no estado isotrópico, existindo regiões com diferentes orientações. Zhang e coautores [21] relataram que a orientação do eixo-b de filmes de PELDB altamente estirados é balanceada nas direções DT e DN, enquanto que para menores estiramentos a orientação do eixo-b é mais expressiva na DN em relação à DT.

Sendo assim, pode-se afirmar que os resultados obtidos neste trabalho estão de acordo com os descritos na literatura e que esse fenômeno, de mudança de estrutura em função do grau de estiramento aplicado, enfatiza que simples variáveis de processo podem resultar em variações morfológicas e, portanto, a estrutura morfológica de filmes de polietileno biaxialmente orientados (processo plano e tubular) é uma função complexa de toda história de deformação e resfriamento experimentada pelo material fundido, além das características intrínsecas do polietileno e, portanto, cada caso precisa ser avaliado individualmente.

Por ensaios de difração de raios-X é possível indicar prováveis alterações da estrutura cristalina do material, assim como determinar o grau de cristalinidade $(\mathrm{Xc})$ das amostras, calculando qual é a proporção de regiões cristalina e não cristalina presente no material. Sabe-se também que o grau de orientação molecular das cadeias poliméricas pode ter influência no perfil do difratograma, o que pode influenciar na metodologia de cálculo do valor de Xc. Portanto, para se ter um valor médio de cristalinidade das amostras estudadas, sem influência do efeito de orientação em uma determinada direção, os difratogramas foram obtidos por reflexão e com as amostras girando, medindo assim a cristalinidade dos cristais orientados nas diferentes direções no plano dos filmes.

O difratograma de raios-X do filme PE-or é apresentado na Figura 3(a). O padrão de difração foi indexado usando a célula unitária ortorrômbica do polietileno com ângulos $\alpha=\beta=\gamma=90^{\circ}$ e parâmetros de rede $\mathrm{a}=0,741 \mathrm{~nm}, \mathrm{~b}=0,494 \mathrm{~nm}$ e $\mathrm{c}=0,255 \mathrm{~nm}$ [27]. São observados dois picos intensos a $2 \theta=21,2^{\circ}$ e $23,4^{\circ}$ correspondentes aos planos de difração (110) e (200) respectivamente, e um pico de baixa intensidade em $\theta=$ $26,2^{\circ}$ correspondente ao plano de difração (210) e uma larga banda amorfa sob esses picos [28]. A deconvolução matemática dos picos também é apresentada na Figura 3(a) e seu resultado indica que o filme PE-or possui Xc de aproximadamente $41 \%$. Dados da literatura [2] sobre cristalinidade de PELBD determinada por calorimetria exploratória diferencial (DSC) indicam grau de cristalinidade entre 22 e 55\%.A Figura 3(b) apresenta os difratogramas de raios-X obtidos para os filmes PE-3, PE-3,5, PE-4 e PE-4,5. Observa-se que esses filmes possuem o mesmo padrão de difração apresentado pelo filme PE-or e, portanto, não houve nenhuma alteração nas dimensões dos eixos da célula unitária do PE devido ao maior grau de estiramento dos filmes na DM, ou seja, a estrutura cristalina foi mantida.

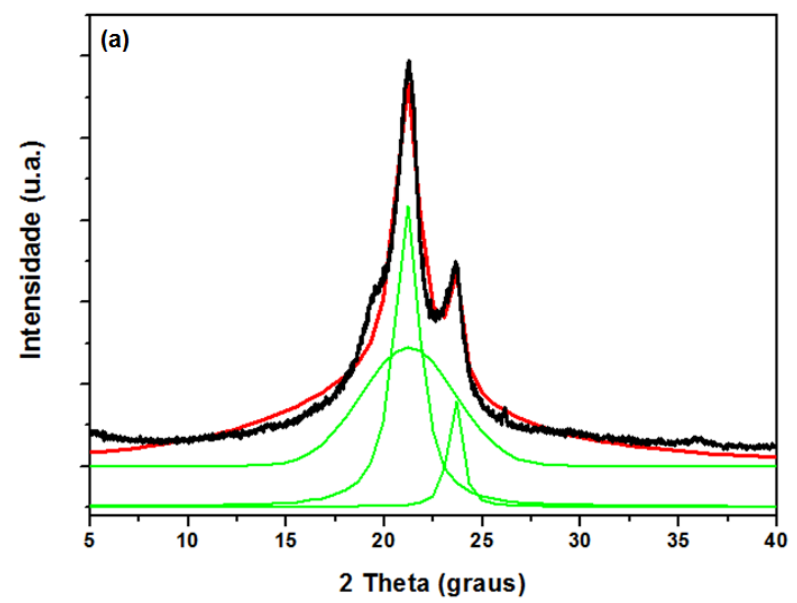




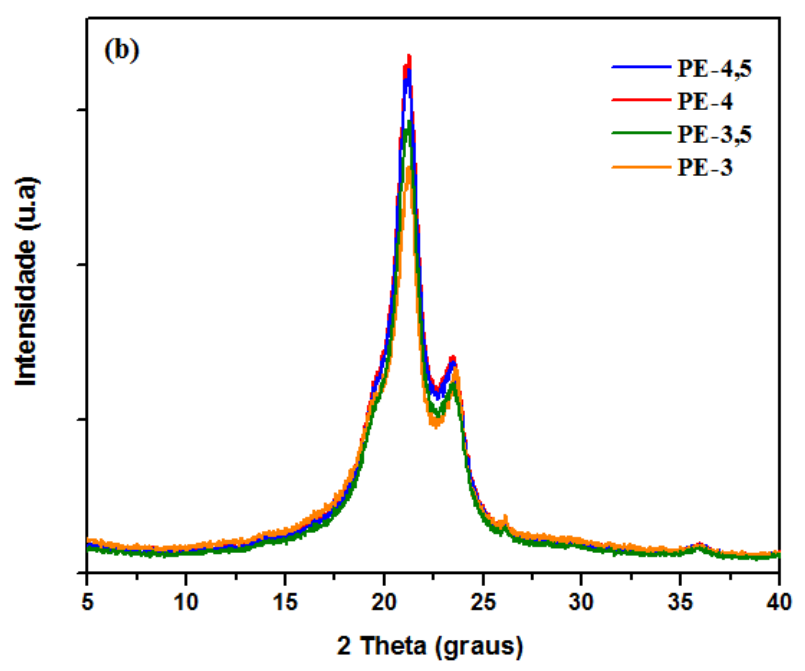

Figura 3: Difratograma de raios-X dos filmes (a) PE-or; e (b) PE-3; PE-3,5; PE-4; PE-4,5.

O grau de cristalinidade calculado por deconvolução dos difratogramas apresentados na Figura 3 é apresentado na Tabela 2. O filme PE-no, não orientado, apresenta baixo grau de cristalinidade, Xc $=17 \%$, como esperado, devido ao tratamento térmico sofrido durante o preparo. O rápido resfriamento do polímero no estado fundido não permite que estruturas organizadas sejam formadas, resultando assim em material com alta fração amorfa.

É conhecido que o processo de estiramento de polímeros semicristalinos, tais como o polietileno, pode aumentar, diminuir ou mesmo não alterar a cristalinidade do polímero. A alteração na cristalinidade depende de vários fatores sendo os principais a estrutura inicial, composição e natureza química do sistema polimérico, e as condições de estiramento como temperatura, razão e velocidade de estiramento e tratamento térmico [29]. Em relação aos filmes PE-3, PE-3,5, PE-4 e PE-4,5, os resultados de cristalinidadeindicam quehouve uma tendência de aumento da cristalinidade dos filmes, considerando os erros das medidas, com o aumento do grau de estiramento, com exceção para o filme PE-4,5 o qual apresentou cristalinidade semelhante à amostra PE-4.

O aumento da cristalinidade é devido ao fato de que o estiramento provoca organização das cadeias moleculares (aumenta a densidade) da fração amorfa do polietileno. O processo de estiramento induz a orientação e o empacotamento das cadeias mais eficientes e ação mais efetiva das forças intermoleculares, aumentando assim a cristalinidade [2]. Esse fenômeno é conhecido na literatura por cristalização induzida por estiramento [4]. Em relação ao filme PE-4,5, para o qual não foi observado aumento da cristalinidade em comparação com o filme PE-4, observa-se certa correlação com os resultados de orientação molecular, pois os valores de $\mathrm{RD}_{730}$ e $\mathrm{RD}_{720}$ obtidos para esses filmes também foram próximos. Esse comportamento se deve, provavelmente, a um limite de alteração da cristalinidade e da orientação molecular em função do grau de estiramento na DM, devido à estrutura molecular do polímero e as condições de processo.

Tabela 2: Grau de cristalinidade $(\mathrm{Xc})$, permeabilidade ao oxigênio $\left(\mathrm{PO}_{2}\right)$ e permeabilidade ao vapor de água $\left(\mathrm{P}_{\mathrm{WV}}\right)$ dos filmes estudados.

\begin{tabular}{l|l|l|l}
\hline AMOSTRA & $\mathbf{X c}(\boldsymbol{\%})$ & $\begin{array}{l}\mathbf{P O}_{\mathbf{2}} \mathbf{x} \mathbf{1 0} \\
\left(\mathbf{c m}^{\mathbf{3}} \mathbf{. c m} / \mathbf{c m}^{\mathbf{2}} \mathbf{. s . c m H g}\right)\end{array}$ & $\begin{array}{l}\mathbf{P}_{\mathbf{W V}} \mathbf{x} \mathbf{1 0}^{\mathbf{3}} \\
\left(\mathbf{c m}^{\mathbf{3}} \mathbf{. c m} / \mathbf{c m}^{\mathbf{2}} \cdot \mathbf{h} \cdot \mathbf{b a r}\right)\end{array}$ \\
\hline PE-or & $41 \pm 3$ & $5,41 \pm 0,01$ & $4,12 \pm 0,52$ \\
\hline PE-3 & $47 \pm 3$ & $5,12 \pm 0,02$ & $3,97 \pm 0,98$ \\
\hline PE-3,5 & $51 \pm 3$ & $4,87 \pm 0,02$ & $3,71 \pm 0,76$ \\
\hline PE-4 & $55 \pm 3$ & $4,48 \pm 0,02$ & $3,43 \pm 0,72$ \\
\hline PE-4,5 & $54 \pm 3$ & $4,43 \pm 0,03$ & $3,39 \pm 0,42$ \\
\hline PE-no & $17 \pm 5$ & $8,52 \pm 0,87$ & $7,12 \pm 1,53$ \\
\hline
\end{tabular}


Em relação à correlação orientação molecular e cristalinidade em filmes de PE, dados da literatura destacam que, em geral, a cristalinidade tende a aumentar com o aumento da orientação das cadeias poliméricas devido ao estiramento [2].O estiramento faz com que ocorra o alinhamento, a orientação molecular e que o empacotamento das cadeias poliméricas seja mais eficiente, assim, forças intermoleculares se tornam mais efetivas, resultando no aumento da cristalinidade do polímero.

Os resultados de permeabilidade ao oxigênio $\left(\mathrm{PO}_{2}\right)$ e de permeabilidade ao vapor de água $\left(\mathrm{P}_{\mathrm{wV}}\right)$ obtidos para os filmes de polietileno estudados foram apresentados na Tabela 2. Esses resultados mostram que, considerando o erro das medidas, a permeabilidade ao oxigênio e ao vapor de água dos filmes tende a diminuir com o aumento do grau de estiramento na DM, e que essa diminuição é pouco expressiva para estiramentos superiores ao sofrido pela amostra PE-4, ou seja, para estiramento com razão DM:DT maior que 4:1.

O comportamento de diminuição, mesmo que discreta, da permeabilidade dos filmes de polietileno com o aumento do grau de estiramento é devido ao fato de que com o estiramento ocorre o alinhamento das cadeias da região amorfa do polímero e, conseqüentemente, ocorre um aumento da cristalinidade do material estirado, aumento do caminho difusional dos permeantes, devido à maior tortuosidade das regiões cristalinas e uma diminuição da permeabilidade. O contínuo estiramento sofrido pelos filmes pode ter atingido o alinhamento máximo suportado naquela direção, o que justifica o fato das propriedades apresentadas pelos filmes PE-4 e PE-4,5 serem semelhantes. Como descrito anteriormente, os resultados de difração de raios-X evidenciaram o aumento discreto da cristalinidade para as amostras PE-or, PE-3, PE-3,5 e PE-4, por outro lado, a amostra PE-4,5 apresentou cristalinidade (e orientação molecular) semelhante à amostra PE-4. Portanto, os resultados de cristalinidade corroboram os resultados de permeabilidade.

A amostra PE-no apresentou alta permeabilidade ao oxigênio e ao vapor de água, como esperado, pois se trata de uma amostra altamente amorfa e não orientada (isotrópica), e, portanto, permeável aos permeantes. A literatura cita valores de permeabilidade ao oxigênio para filmes isotrópicos de PELBD na faixa de 6,6 a $9,8 \times 10^{-10} \mathrm{~cm}^{3} . \mathrm{cm} / \mathrm{cm}^{2}$.s.cmHg [30]. Não foram encontrados na literatura dados de permeabilidade ao vapor de água para amostra isotrópica.

Os demais filmes estirados apresentaram valores de permeabilidade de acordo com a literatura, a qual descreve valores de permeabilidade ao oxigênio de $4,1 \times 10^{-10} \mathrm{~cm}^{3} . \mathrm{cm} / \mathrm{cm}^{2} . \mathrm{s} . \mathrm{cmHg}$ [31] e de permeabilidade ao vapor de água de $14,8 \times 10^{-3}$ [32] e $3,8 \times 10^{-3} \mathrm{~cm}^{3} . \mathrm{cm} / \mathrm{cm}^{2}$.h.bar [33] para filmes de PELBD.

Os principais fatores que afetam a permeabilidade ao oxigênio e ao vapor de água de filmes de polietileno são a densidade (indicada pelo grau de cristalinidade), a orientação molecular da fase cristalina do polímero e a espessura do filme. A permeabilidade de filmes de PELBD pode aumentar significativamente com a redução da densidade da resina e com a redução da espessura do filme, devido à menor tortuosidade [2, 13, 34]. A permeabilidade deve ser menor em filmes que possuem orientação cristalina, a qual gera caminhos mais tortuosos ao permeante. Estudos têm demonstrado com sucesso a correlação da orientação molecular cristalina com a permeabilidade em filmes de PELBD. Uma grande diminuição da permeabilidade a gases [30] e a vapores orgânicos [35] em filmes de PELBD foi observada com o aumento do grau de estiramento do filme. Essa redução foi interpretada como conseqüência do empacotamento denso, devido à organização de regiões amorfas, produzido pelo processo de estiramento.

À luz do conceito de tortuosidade e permeabilidade das lamelas aos permeantes, pode-se afirmar que o arranjo ideal de lamelas para se ter melhores propriedades de barreira, seria com a normal das lamelas paralela à superfície do filme de polietileno [19]. No entanto, os resultados mostram que a realização de tal morfologia não é possível, pois os filmes de polietileno cristalizam segundo o modelo de Keller e Machin. As tentativas de produzir uma orientação mais equilibrada através de alterações das variáveis de processo para produzir diferentes quantidades de orientação dos eixos- a e -c, em relação às direções dos estiramentos (DM e/ou DT) no plano do filme, vêm sendo amplamente estudadas. No entanto, devido ao fato do eixo de crescimento do cristal de polietileno ser ao longo do eixo-b, que se orienta perpendicular à direção da tensão aplicada, nenhuma quantidade de estiramento, seja na DT ou na DM, detém o crescimento lamelar perpendicular à superfície do filme. Assim, mesmo que uma quantidade de orientação do eixo-b em relação às direções DM e DT possa ser alterada, haverá uma inerente quantidade de cadeias ao longo do eixo-b que irá permanecer orientada em relação à DN. Embora o modelo de Keller-Machin não faça qualquer previsão em relação ao estado de orientação das cadeias da fase amorfa, pode-se afirmar que esse estado tem alguma influência no comportamento de permeabilidade de gases e vapor de água em filmes de polietileno. Entretanto, a literatura existente descreve resultados conflitantes, não descritos nesse trabalho. Sendo assim, os resultados desse trabalho demonstraram o efeito do caminho tortuoso e a influência de altos níveis de orientação das fases cristalina e amorfa no processo de permeação em filmes de PELBD. 


\section{CONCLUSÕES}

A partir das caracterizações de orientação molecular realizadas foi possível observar que houve a troca da orientação molecular cristalina nos filmes de PELBD em função do grau de estiramento dos filmes. O filme com baixo grau de estiramento apresentou arranjo das lamelas com eixo-a orientado paralelo à DM (direção do estiramento) e eixos-b e -c orientados perpendicular à DM (estrutura Keller-Machin I ou textura-a). Por outro lado, os filmes com alto grau de estiramento apresentaram estrutura na qual o eixo-c da estrutura se orienta paralelamente à DM e os eixos-a e -b da célula unitária se orientam paralelamente e de forma balanceada nas direções DT e DN (estrutura Keller-Machin II ou textura-c).

A caracterização do grau de cristalinidade evidenciou a tendência de aumento do grau de cristalinidade em função do maior grau de estiramento dos filmes, indicando assim, certa correlação entre orientação molecular, induzida devido ao estiramento, e cristalinidade dos filmes de PE.

Os resultados de propriedades de barreira mostraram que tanto a permeabilidade ao oxigênio, como a permeabilidade ao vapor de água dos filmes de PE tendem a diminuir com o aumento do grau de estiramento na DM. É importante destacar que houve uma tendência de diminuição dessas propriedades, pois considerando os erros das medidas, muitas vezes os resultados obtidos permaneceram sem grandes alterações. Os resultados de grau de cristalinidade e de permeabilidade se relacionam entre si, através da observação do aumento do caminho difusional dos permeantes, devido à maior tortuosidade das regiões cristalinas e, consequente, diminuição da permeabilidade. Em relação à correlação permeabilidade e orientação molecular, este estudo, assim como outros da literatura, mostra que a orientação devido ao estiramento, pode realmente diminuir a taxa de permeação, no entanto, o arranjo ideal das lamelas para se ter melhores propriedades de barreira não é possível devido à forma de cristalização do polímero.

Sendo assim, pode-se afirmar que a razão de estiramento é uma variável de processo que influencia fortemente a microestrutura final do filme de PELBD, a cristalinidade e as propriedades de permeação ao vapor de água e ao oxigênio. Os resultados também evidenciaram que existe um valor limite de razão de estiramento na $\mathrm{DM}$, a partir do qual não é evidenciada alterações na cristalinidade e permeabilidade dos filmes.

\section{AGRADECIMENTOS}

Ao CNPq (309107/2013-0) pelo suporte financeiro. À Profa. Y. P. Mascarenhas pelo uso do difratômetro de raios-X. Ao Prof. A. L. Pessan pelo uso do espectrofotômetro de absorção de infravermelho.

\section{BIBLIOGRAFIA}

[1] SCHOUTERDEN, P., GROENINCKX, G., VAN DER HEIJDE, B., et al., "Fractionation and thermal behaviour of linear low density polyethylene", Polymer, v. 28, pp. 2099-2104, 1987.

[2] PEACOCK, A. J. Handbook of Polyethylene: structures, properties and applications. New York: Marcel Dekker Inc., 2000.

[3] SUKHADIA, A. M., DATTA, A., BAIRD, D. G. "Mixing history on the morphology and properties of thermoplastic/LCP blends", InternationalPolymerProcess, v. 7, pp. 218-228, 1992.

[4] MANRICH, S. Processamento de Termoplásticos: rosca única, extrusão e matrizes, injeção e moldes. São Paulo, ArtliberEditora, 2005.

[5] COLE, K. C, AJJI, A. Characterization of orientation.In: Ward, I. M., Coates, P.D., Dumoulin, M.M., editors. Solid phase processing of polymers. Munich, Carl HanserVerlag, 2000.

[6] CHOI, K., SPRUIELL, J. E., WHITE, J. L. "Orientation and morphology of high-density polyethylene film produced by the tubular blowing method and its relationship to process conditions", Journal of Polymer Science Polymer Physics Edition, v. 20, pp. 27-47, 1982.

[7] BRANCIFORTI, M. C., PIMENTEL, R., BERNARDI, A., et al., "Correlations between processing parameters, morphology, and properties of blown films of linear low-density polyethylene/low-density polyethylene blends. Part I. Crystalline biaxial orientation by IR and mechanical properties", .Journal of Applied Polymer Science, v. 101, pp. 3161-3167, 2006.

[8] BRANCIFORTI, M. C., GUERRINI, L. M., MACHADO, R., et al., "Correlations between processing parameters, morphology, and properties of blown films of LLDPE/LDPE blends, part II: Crystalline and amorphous biaxial orientation by WAXD pole figures", Journal of Applied Polymer Science, v. 102, pp. 2760-2767, 2006. 
[9] KIDA, T., OKU, T., HIEJIMA, Y., et al., "Deformation mechanism of high-density polyethylene probed by in situ Raman spectroscopy”, Polymer, v. 58, pp. 88-95, 2015.

[10] KIDA, T., HIEJIMA, Y., NITTA, K-H. "Molecular orientation behavior of isotactic polypropylene under uniaxial stretching by rheo-Raman spectroscopy”, Polymer Letters, v. 10, pp. 701-709, 2016.

[11] KELLER, A.; MACHIN, M. J. "Oriented crystallization in polymers". Journalof Macromolecular Science Physics B, v. 1, pp. 41-91, 1967.

[12] SARANTÓPOULOS, C. I. G. L., OLIVEIRA. L. M., PADULA, M., et al., Embalagens plásticas flexíveis: principais polímeros e avaliação de propriedades. Campinas: CETEA/ITAL editora, 2002.

[13] SIRACUSA, V. "Food Packaging Permeability Behaviour: A Report", International Journal of Polymer Science, v. 2012, pp. 1-11, 2012.

[14] ASHLEY, R. J. "Permeability and Plastics Packaging, In: Polymer Permeability”, J. Comyn, ed., Barking: Chapman@ Hall, 1985.

[15] ASTM D3985-05, Standard Test Method for Oxygen Gas Transmission Rate Through Plastic Film and Sheeting Using a Coulometric Sensor, ASTM International, West Conshohocken, PA, 2010.

[16] ASTM F1249-13, Standard Test Method for Water Vapor Transmission Rate Through Plastic Film and Sheeting Using a Modulated Infrared Sensor, ASTM International, West Conshohocken, PA, 2013.

[17] PERRY, R. H., GREEN, D. Perry's Chemical Engineer's Handbook. New York, McGraw Hill, 1997.

[18] MADDAMS, W. F., PREEDY, J. E. "X-ray diffraction orientation studies on blow polyethylene films. III. High-stress crystallization orientation”, Journal of Applied Polymer Science, v. 22, pp. 2751-2759, 1978.

[19] SCHLOTTER, N. E., FURLAN, P. Y. “A review of small molecule diffusion in polyolefins", Polymer, v. 33, pp.3323-3342, 1992.

[20] AJJ, A., AUGER, J., HUANG, J., et al., "Biaxial stretching and structure of various LLDPE resins", Polymer Engineering \& Science, v. 44, pp. 252-260, 2004.

[21] ZHANG, X. M., ELKOUN, S., AJJI, A., et al., "Oriented structure and anisotropy properties of polymer blown films: HDPE, LLDPE and LDPE”, Polymer, v. 45, pp. 217-229, 2004.

[22] LU, J., SUE, H. J., RIEKER, T. P. "Morphology and mechanical property relationship in linear lowdensity polyethylene blown films", Journal of Materials Science, v. 35, pp. 5169-5178, 2000.

[23] KWACK, T. H., HAN, C. D. "Development of crystalline structure during tubular film blowing of lowdensity polyethylene”, Journal of Applied Polymer Science, v. 35, pp. 368-389, 1988.

[24] PAZUR, R. J., PRUD'HOMME, R. E. “X-ray pole figure and small angle scattering measurements on tubular blown low-density polyethylene”, Macromolecules, v. 29, pp. 119-128 1996.

[25] YU, T. H., WILKES, G. L. "Orientation determination and morphological study of high density polyethylene (HDPE) extruded tubular films:Effect of processing variables and molecular weight distribution”, Polymer, v. 37, pp. 4675-4687, 1996.

[26] JOHNSON, M. B., WILKES, G. L., SUKHADIA, A. M., et al., "Optical properties of blown and cast polyethylene films: Surface versus bulk structural considerations", Journal of Applied Polymer Science, v. 77, pp. 2845-2864, 2000.

[27] BUNN, C. W. "The crystal structure of long-chain normal paraffin hydrocarbons. The shape of the $\mathrm{CH}_{2}$ group”, Transactions of the Faraday Society, v. 35, pp. 482-491, 1939.

[28] LAFRANCE, C-P., DEBIGERÉ, J., PRUD'HOMME, R. E. "Study of crystalline orientation in drawn ultra-high-molecular weight polyethylene films", Journal of Polymer Science Part B: Polymer Physics, v. 31, pp. 255-264, 1993.

[29] VASANTHAN, N., SALEM, D. R. "FTIR spectroscopic characterization of structural changes in polyamide-6 fibers during annealing and drawing”, Journal of Polymer Science Part B: Polymer Physics, v. 39, pp. 536-554, 1997.

[30] HOLDEN, P. S., ORCHARD, G. A. J., WARD, I. M. “A study of the gas barrier properties of highly oriented polyethylene”, Journal of Polymer Science: Polymer Physics Edition. v. 23, pp. 709-731, 1985.

[31] WARD, I. M. Structure and Properties of Oriented Polymers. London, Chapman and Hall, 2nd ed, 1997.

[32] KAMAL, M. R., JINNAH, I. A., UTRACKI, L. A. "Permeability of oxygen and water vapor through polyethylene/polyamide films", PolymerEngineering \& Science, v. 24, p. 1337-1347, 1984. 
[33] SILVA, E. M., GORETTI, M., COUTINHO, F., et al., "Influência da concentração e purificação da argila na estrutura e permeação ao vapor de água de nanocompósitos PEBDL/bentonita”, Polímeros: Ciência e Tecnologia, v. 23, pp. 108-114, 2013.

[34] KROHN, J., TATE, R., JORDY, D. "Factors affecting the permeability of PE blown films", Journal of Plastic Film \& Sheeting, v. 13, pp. 327-335, 1997.

[35] PETERLIN, A., WILLIAMS, J. L. "Influence of compacting pressure on the permeability of vectorized membranes for liquids", Journal of Applied Polymer Science, v. 15, pp. 1493-1505, 1971. 